\title{
Recién nacidos pequeños para la edad gestacional: sensibilidad del diagnóstico y su resultado
}

\author{
Lucía Díaz¹, Patricia Quiñones ${ }^{1}$, Diego Vargas², Francisco Cóppola ${ }^{3}$
}

\section{RESUMEN}

La restricción del crecimiento intrauterino (RCIU) se asocia a morbi-mortalidad perinatal, ofrece dificultades diagnósticas y los beneficios de éste, deben ser balanceados con el aumento del intervencionismo.

Objetivo: Determinar la sensibilidad en el diagnóstico clínico-ecográfico de pequeños para la edad gestacional (PEG) y valorar el impacto del diagnóstico prenatal en la tasa de cesáreas y los resultados neonatales (Apgar y gasometría).

Material y Métodos: Estudio descriptivo-analítico retrospectivo.

Resultados: De 4548 nacidos vivos, se detectó un total de 379 PEG; 99 casos (26\%) tenían diagnóstico previo de RCIU. Los resultados neonatales de depresión, acidosis y Apgar bajo no fueron diferentes en el grupo de RCIU comparado con el de sin diagnóstico. La tasa de cesárea fue de $56 \%$ en el grupo de RCIU contra $21 \%$ en el grupo con crecimiento normal. En la población con diagnóstico prenatal, el riesgo de interrupción por cesárea fue de RR =2,64 IC 95\% (1,98-3,51).

Conclusión: Dado el alto porcentaje de embarazos sin control o mal control obstétrico, no se puede concluir sobre la sensibilidad clínica y/o ecográfica del diagnóstico prenatal de RCIU, ni su impacto en los resultados neonatales. El diagnóstico de RCIU aumenta el intervencionismo obstétrico y la morbilidad materna al triplicar el índice de cesáreas. (Horiz Med 2014; 14(2): 6-10)

Palabras clave: Pequeño para la edad gestacional, restricción del crecimiento intrauterino, control prenatal. (Fuente: DeCS BIREME).

\section{Small for gestational age: Sensibility diagnosis and result}

\begin{abstract}
Intrauterine growth restriction (IUGR) is associated with perinatal morbidity and mortality. Presents diagnostic difficulties and benefits should be balanced with the increase of interventions.

Objective: To determine the sensitivity of clinical - ultrasound diagnosis of Small for Gestational Age Infants (SGA). To assess the impact of prenatal diagnosis in cesarean section rate and neonatal outcome.

Material and Methods: Retrospective descriptive and analytical trial, of the Latin American Center of Perinatology database Perinatal Information System (SIP). From March through September 2010 of a total of 4548 newborn child at the Centro Hospitalario Pereira Rossell, 379 were SGA Infants (defined as growth below p10 for gestational age). Of the target population, it was studied whether they had prenatal clinic and ultrasonographic diagnosis, number of prenatal visits, route of delivery and neonatal outcome (Apgar score, blood gases).

Results: Of the 379 SGA cases, $99(26 \%)$ had previous diagnosis of IUGR. The average gestational age for the diagnosis was 35 weeks. Of these 99 diagnosed cases, $88(89 \%)$ had more than 5 prenatal visits compared to the group without previous diagnosis. 190 SGA (68\%) had well controlled pregnancies. There was no difference in the neonatal outcome of depression, acidosis and low Apgar between the group with IUGR or without diagnosis. Cesarean sections were $56 \%$ in the group with previous diagnosis versus $21 \%$ in the group without diagnosis. The population group with prenatal diagnosis had 2,64 RR of cesarean section $(95 \% \mathrm{Cl} 1,98-3,51) \mathrm{p}<0,0001$.

Conclusion: Because of the high percentage of pregnancies uncontrolled or poorly obstetric care can not conclude on the clinical sensitivity and / or prenatal ultrasound diagnosis of IUGR, and its impact on neonatal outcomes. The diagnosis of IUGR increases obstetric interventions and maternal morbidity due to the high rate of cesarean sections.
\end{abstract}

(Horiz Med 2014; 14(2): 6-10)

Key words: Small for gestational age, fetal growth retardation, prenatal care. (Source: MeSH NLM).

Residente de Ginecotocología. Clínica Ginecotocológica “A”. Prof. Dr. L. Briozzo. FMH. Universidad de la República. Uruguay. Asistente de Ginecotocología. Clínica Ginecotocológica “A”. Prof. Dr. L. Briozzo. FMH. Universidad de la República. Uruguay. Profesor Agregado de Clinica Ginecotocològica “A”. Prof. Dr. L. Briozzo. FMH. Universidad de la República. Uruguay 


\section{INTRODUCCIÓN}

Los fetos identificados como pequeños para la edad gestacional (PEG) constituyen un grupo heterogéneo desde el punto de vista de la etiología, conducta y pronóstico $(1,2)$. Se definen como PEG, los fetos con un peso fetal inferior al percentil 10 para una edad gestacional específica $(3,4)$.

Según su etiología, podemos clasificar los fetos PEG en varios grupos (5-7):

PEG constitucional (50- 75\%). Sin alteraciones estructurales, líquido amniótico normal, Döppler de la arteria umbilical normal y velocidad de crecimiento normal.

PEG anómalo (5-10\%). Con anomalías genéticas, estructurales 0 secundarias a infección. Corresponden a los tradicionalmente llamados RCIU simétricos $(8,9)$.

Restricción del crecimiento intrauterino (RCIU) (10$15 \%)$. Se define como una falla del feto en alcanzar su óptimo potencial de crecimiento. Es un grupo heterogéneo asociado a hipertensión, tabaco, infecciones, desnutrición y factores desconocidos o inexplicables. una función placentaria alterada, identificada por Doppler anómalo de la arteria umbilical o reducción de la velocidad de crecimiento $(10,11)$.

Por definición, un feto con RCIU, es aquél que tiene una limitación del potencial de crecimiento intrínseco, se refiere a un patrón de crecimiento en un periodo de tiempo, en cambio el término PEG se refiere a un punto en una curva de peso.

Comparados con los fetos que se desarrollan de forma adecuada, los RCIU presentan mayores tasas de mortalidad, asfixia al nacimiento, hipotermia, hipoglucemia, aspiración de meconio y secuelas neurológicas. Se estima que los fetos con RCIU representan el $40 \%$ de los mortinatos de causa no explicada, el $30 \%$ de los fallecidos por muerte súbita y tienen una mortalidad infantil 8 veces mayor que los fetos con un peso adecuado para su edad de gestación. Además, según la hipótesis de Barker, están expuestos en la época adulta a enfermedades como hipertensión, ateromatosis o intolerancia a la glucosa y síndrome metabòlico (12).
Los métodos empleados para diagnosticar los PEG incluyen: impresión clínica del tamaño fetal mediante palpación abdominal, la medición de la altura uterina, la biometría por ecografía, estimación ecográfica del peso fetal y el estudio de las ondas de velocidad de flujo anómalas de las arterias uterinas (Döppler).

Es fundamental al considerar estas variables un correcto cálculo de la edad gestacional.

El objetivo del presente estudio fue determinar la sensibilidad en el diagnóstico clínico-ecográfico de PEG en la Maternidad del Centro Hospitalario Pereira Rossell (CHPR).

\section{MATERIAL Y MÉTODOS}

\section{Estudio descriptivo y analítico retrospectivo.}

Se revisó la base del datos del Sistema Informático Perinatal (SIP), desarrollado por el Centro Latinoamericano de Perinatología (CLAP) desde el primero de marzo al 30 de setiembre de 2010. Se registraron 4548 nacidos vivos, de los cuales 379 eran PEG; (según la variable definida en el SIP “peso según EG") .

\section{Criterios de inclusión: Todos los RN PEG.}

Criterios de exclusión:

- RN adecuados para la edad gestacional (AEG)

- RN entre p10 y 90 para la edad gestacional

- RN grandes para la edad gestacional (GEG)

- RN con peso por encima del p90 para la edad gestacional y

- Embarazos múltiples.

Se realizó relevamiento de las historias de recién nacidos PEG en el archivo del Hospital de la Mujer de los nacimientos en el período de estudio. Se realizó auditoría de cada una de ellas buscando variables en estudio

\section{Definiciones y variables}

PEG: recién nacidos con peso menor al percentil 10 para su edad gestacional según tablas poblacionales de crecimiento de peso y edad gestacional. 
DIAGNÓSTICO CLÍNICO RCIU : Altura uterina e impresión clínica del tamaño fetal. La valoración del tamaño uterino constituye todavía el método más común en el despistaje del RCIU debido a su disponibilidad, reproducibilidad, simplicidad y bajo costo (2).

DIAGNÓSTICO ECOGRÁFICO: Ecográficamente la mejor estimación del tamaño fetal la da la circunferencia abdominal (CA) y el cálculo del peso fetal $(2,10,13,14)$. Puesto que el proceso de crecimiento es dinámico, una valoración seriada de los parámetros fetales (separados 14-21días), nos proporcionarán una mayor información (14). Para nuestro estudio se consideró diagnóstico de RCIU al menos uno de los criterios clínicos y/o ecográficos mencionados.

GASOMETRÍA: La toma de sangre de la arteria umbilical se realizó en forma inmediatamente posterior al nacimiento con previo doble clipado del cordón umbilical. Se analizaron los parámetros del ph y exceso de bases tomando como punto de corte para diagnóstico de acidosis un $\mathrm{ph} \leq 7,1 \mathrm{y}$ $\mathrm{EB} \leq 10$ (15).

APGAR: Método desarrollado por la Dra. V. Apgar para evaluar la adaptación del recién nacido a la vida extrauterina.

Se evalúan 5 ítems, frecuencia cardíaca, esfuerzo respiratorio, tono muscular, color, llanto, al minuto y a los cinco minutos con una escala del 0-2. Un score de 0-3 representa depresión severa, 4-7 indica depresión moderada y un score $8-10$ se considera como una buena adaptación a la vida extrauterina.

Se tomó como punto de corte en el estudio un Apgar a los cinco minutos igual o menor a 7

VIA DE FINALIZACIÓN DEL EMBARAZO: dividiéndose en dos grupos, vía vaginal (incluye parto instrumental) y operación cesárea. No se analizó la oportunidad de la finalización (emergencia, urgencia, coordinación).

Los datos obtenidos fueron ingresados en planilla Excel y su procesamiento se realizó mediante programa estadísticos.

\section{RESULTADOS}

El total de RN en la base SIP en el período analizado (enero-setiembre 2010) fue 4548; PEG 379 , (8,33\%); lo cual es coincidente con las cifras publicadas a nivel nacional e internacional (13). Los AEG fueron $3841(84,45 \%)$ y GEG correspondieron a $280(6,15 \%)$. En 48 casos $(9,48 \%)$, había una falta del llenado del SIP con respecto a la clasificación según peso/ edad gestacional.

De los 379 casos analizados, 99 casos $(26,12 \%)$ tenían diagnóstico previo de RCIU. La edad gestacional promedio al diagnóstico fue de 35 semanas, siendo la EG promedio al nacimiento 37 semanas.

Del total de RCIU, 88 casos $(88,89 \%)$ habían sido embarazos con más de 5 consultas prenatales, en comparación con el grupo sin diagnóstico previo en donde 175 casos $(66.02 \%)$ fueron embarazos bien controlados.

Tabla 1. Características de la población

\begin{tabular}{cc}
\hline Característica & Promedio \\
\hline Edad (años) & 27 \\
Paridad & 1.76 \\
Partos vaginales & 103 \\
Cesáreas & 276 \\
$\geq 5$ controles & 263 \\
$>5$ controles & 42 \\
Sin control & 59 \\
\hline
\end{tabular}

Al considerar las causas que influyeron en la falta del diagnóstico previo de RCIU se desprende que en 7 casos $(2.48 \%)$, hubo una discordancia de $+/$ 2 semanas entre la EG obstétrica calculada por gestograma del CLAP a partir de FUM confiable y/o ecografía del primer trimestre y la EG neonatal mediante método Capurro. 
En 10 casos (3.56\%), se constató en las consultas prenatales una AU discordante en menos con la EG, sin realizarse sin embargo diagnóstico clínico, ni solicitarse la paraclínica pertinente.

En 57 casos (20.23\%), fueron embarazos sin control obstétrico.

En 33 casos (11.71\%) correspondieron a embarazos con un mal control, definido por la OMS como menos de 4 consultas prenatales (16).

En 175 casos (62.02\%) fueron embarazos bien controlados, clínica-ecográfico, sin realizarse diagnóstico prenatal.

En cuanto a la vía de finalización de la gravidez, en aquellos con diagnóstico prenatal de RCIU, el $55,56 \%$ (55) fue mediante operación cesárea (CST) y el 44,44\% (44) por parto vaginal (PV); incluyendo el parto instrumental; en comparación con aquellos nacimientos sin diagnóstico previo en que la CST sólo constituyó el $21.07 \%$ de la vía de finalización y el PV el restante $78,93 \%$.

En cuanto a los resultados obstétricos teniendo en cuenta el Apgar y la gasometría, no hay diferencias significativas entre ambos grupos; $\mathrm{pH} 7.25$, BE -3.588, Apgar $8 / 9$ en fetos con diagnóstico vs $\mathrm{pH} 7,29, \mathrm{BE}-4.441$, Apgar 8/9.

Tabla 2. Resultados según presencia a ausencia de diagnóstico prenatal

\begin{tabular}{ccc}
\hline & $\begin{array}{c}\text { Con diagnóstico } \\
\text { prenatal }\end{array}$ & $\begin{array}{c}\text { Sin diagnóstico } \\
\text { prenatal }\end{array}$ \\
\hline 25 controles & $88(89 \%)$ & $190(68 \%)$ \\
$<5$ controles & $9(9 \%)$ & $33(12 \%)$ \\
sin control & $2(2 \%)$ & $57(20 \%)$ \\
Parto vaginal & $44(44 \%)$ & $221(89 \%)$ \\
Cesárea & $55(56 \%)$ & $59(21 \%)$ \\
Depresión neonatal (Apgar $>7)$ & $4(4 \%)$ & $19(7 \%)$ \\
Acidosis (pH < 7.1 BE <-10) & $5(5 \%)$ & $8(3 \%)$
\end{tabular}

\section{DISCUSIÓN}

Todas las mujeres deben ser evaluadas independientemente de sus factores de riesgo para identificar un PEG.

Las mujeres que tienen tres o más factores de riesgo de menor importancia deben ser referidas para estudio Doppler de la arteria uterina entre las 20-24 semanas de gestación.

Las mujeres con un Doppler de las arterias uterinas normales no requieren la medición en serie del tamaño del feto y tampoco seguir con una evaluación del bienestar con Doppler de la arteria umbilical a menos que desarrollen durante el embarazo complicaciones, por ej emplo: hemorragia preparto o hipertensión. Sin embargo, se les debe ofrecer una exploración para el tamaño del feto y el Doppler de la arteria umbilical durante el tercer trimestre $(2,5)$.

El Apgar y la gasometría, no fueron de mucha ayuda en nuestro estudio. Sin embargo, no se descarta su indicación $(9,15,16)$.

Un feto con RCIU, refleja una alteración en el desarrollo dependiente del tiempo por lo que debe diferenciarse de manera adecuada del PEG lo que permitirá vislumbrar el manejo y pronóstico.

En conclusión, dado el alto porcentaje de embarazos sin control obstétrico (20\%) o con un mal control obstétrico $(11,7 \%)$ no se puede concluir que exista una baja sensibilidad en el diagnóstico prenatal de RCIU y por tanto inferir el impacto de éste sobre los resultados perinatales.

Del presente estudio, se destaca la influencia que tiene el conocimiento por parte del equipo médico de aquellos embarazos complicados con RCIU, ya que su diagnóstico por sí mismo triplica el índice de cesáreas, aumentando por tanto el intervencionismo obstétrico y la morbilidad materna. 


\section{Fuentes de financiamiento \\ Autofinanciado por los autores.}

\section{Conflictos de interés}

Los autores declaran no tener ningún conflicto de interés.

\section{REFERENCIAS BIBLIOGRÁFICAS}

1. Manning FA. General principles and applications of ultrasonography. In Creasy RK, Resnik R, (eds). Maternal-Fetal Medicine: Principles and Practice. Philadelphia: Saunders, 2004.

2. RCOG Guideline No. 31. The investigation and management of the small for gestational age fetus. November 2013. Available at: www. rcog. org. uk.

3. Garite TJ, Clark R, Thorp JA. Intrauterine growth restriction increases morbidity and mortality among premature neonates. Am J Obstet Gynecol 2004; 191:481-7.

4. Resnik R. Intrauterine growth restriction. Obstet Gynecol 2002; 99:490-6.

5. Bobrow CS, Soothill PW. Fetal growth velocity: a cautionary tale. Lancet 1999; 353:1460.

6. Tan TY, Yeo GS. Intrauterine growth restriction. Curr Opin Obstet Gynecol 2005; 17:135-42.

7. Divon MY, Chamberlain PF, Sipos L, Manning FA, Platt LD. Identification of the small for gestational age fetus with the use of gestational age-independent indices of fetal growth. Am J Obstet Gynecol 1986; 155:1197-201.

8. Resnik R, Creasy RK. Intrauterine growth restriction. In Creasy RK, Resnik R. (eds). Maternal-Fetal Medicine: Principles and Practice. Philadelphia: Saunders, 2004.

9. Brodsky D, Christou H. Current concepts in intrauterine growth restriction. J Intensive Care Med 2004; 19:307-19.

10. Baschat AA. Fetal growth disorders in high risk pregnancies management options. James DK, Steer PJ, Weiner CP, Gonik B (eds). Saunders Elsevier (Publ). Philadelphia, 2006. Chap 12. pp 240-71.
11. Ville Y, Nyberg DA. Growth, Doppler and fetal assessment. In: Nyberg DA, McGahan JP, Petrorius DH, Pilu G. (eds). Diagnostic imaging of fetal anomalies. Philadelphia: Lippincot, Willians \& Wilkins; 2003. pp 31-58.

12. Luckas M. Fetal growth restriction in Obstetrics and Gynecology. An evidence-based text for MRCOG. Luesley DM, Baker PN (eds). Arnold (Publ). London 2004. pp 245-52.

13. Shah PS, Zao J,Ali S. Maternal marital status and birth outcomes: a systematic review and meta-analyses. Matern Child Health J 2011; 15:1097-109.

14. Haram K, Søfteland E, Bukowski R. Intrauterine growth restriction. Int J Gynecol Obstet 2006;93:5-12.

15. Briozzo L, Sosa Fuertes C, Martínez A, Rodríguez F, Ferreiro G, Panizza R, et al. Análisis de la prevalencia de sufrimiento fetal agudo, asfixia perinatal y síndrome hipóxico isquémico en la maternidad del Centro Hospitalario Pereira Rossell. Arch Gin Obstet 2003; 41(3): 84-91.

16. Villar J et al. For the WHO Antenatal Care Trial Research Group. WHO antenatal care randomized trial for the evaluation of a new model of routine antenatal care. Lancet 2001, 357: 1551-1564.

\section{Correspondencia:}

Francisco Cóppola

Dirección: Clínica Ginecotocológica A Prof. Dr. Leonel Briozzo, Facultad de Medicina de la Universidad de la República. Uruguay

Teléfono: + 59894451100

Correo electrónico: fracoop60@yahoo.es 